\title{
IRAS LRS Spectral Class and Light Curve of M \& S Miras
}

\author{
M.S. Vardya
}

Tata Institute of Fundamental Research

Homi Bhabha Road, Bombay 400 005, India

A large sample of 177 Miras, comprising $164 \mathrm{M}$ and $13 \mathrm{~S}$ stars, has been examined to determine the dependence of $9.7 \mu \mathrm{m}$ silicate emission, as revealed by their IRAS LRS Spectral class, on the visual light curve asymmetry factor, $f$. It is found that the silicate feature occurs not only in M (Vardya et al. 1986; Onaka \& de Jong 1987) but in S Miras also only for $f \leq 0.45$. This, however, is only a necessary condition, as about one fifth of Miras with $\mathrm{f} \leq 0.45$ do not show the $9.7 \mu \mathrm{m}$ emission. This non-detection shows dependence on other parameters like the mean visual light amplitude. Non-detection is highest in the region $0.43<f$ $\leq 0.45$, as well as when mean amplitude is $\leq 5^{\mathrm{m}} .0$. Though strong emission features in M Miras may occur for any value of $f$, very weak features are absent for small values of $f$, and the strongest feature tends to appear for large values of $f$. Infrared excess tends to increase with increase in the strength of the silicate emission and with decrease in the value of $f$.

Detection of silicate emission, viewed from the visual light curve classes (Ludendorff, 1928) is very high for $\alpha_{1}, \alpha_{2}$, and $\alpha_{3}$ classes, decreases for $\alpha_{4}$ and $\gamma_{1}$, and is negligible for $\beta$ class. The strength of the silicate emission is highest for the $\alpha_{1}$ class, decreases for $\left(\alpha_{2}, \alpha_{3}, \alpha_{4}\right)$ - classes, and is the lowest for the $\gamma_{1}$ class.

Coming to the $\mathbf{S}$ Miras, it is surprising that not a single $S$ star shows a strong silicate feature, when even a C Mira, RV Cen, shows it. This may reflect a gradual change from $M$ to $S$ phase. This may also be due to silicate emission peak being somewhat shif ted redward from $9.7 \mu \mathrm{m}$.

The above results can be understood qualitatively. However, a quantitative treatment of pulsation, shock waves, and condensation chemistry is essential for proper application.

Ref erences:

Lundendorff. H. 1928, Handbuch der Astrophysik, Springer Verlag: Berlin, vol. 6, chap. 2, p. 49 .

Onaka, T. and de Jong, T. 1987, Late Stages of Stellar Evolution, eds. S. Kwok and S.R. Pottasch, D. Reidel, Dordrecht, p. 97.

Vardya, M.S., de Jong, T., and Willems, F.J. 1986, Astrophys. J. 304, L29. 